Check for updates

Cite this: Nanoscale Adv., 2019, 1, 273

\title{
Sub-picogram level sensitivity in HIV diagnostics achieved with the europium nanoparticle immunoassay through metal enhanced fluorescence
}

\author{
Aditya Kurdekar, (D) a L. A. Avinash Chunduri, (D) ab Mohan Kumar Haleyurgirisetty, ${ }^{c}$ \\ Indira K. Hewlett ${ }^{\mathrm{C}}$ and Venkataramaniah Kamisetti (D) *a
}

\begin{abstract}
We describe a novel application of Metal Enhanced Fluorescence (MEF) to immunoassays for boosting the signal through a single step modification of the europium nanoparticle based immunoassay with addition of gold nanoparticles. The new limit of detection was found to be $0.19 \mathrm{pg} \mathrm{mL}^{-1}$ which was much lower than that of the conventional assay which was around $1.80 \mathrm{pg} \mathrm{mL}^{-1}$, thus achieving a ten-fold increase in the limit of detection of p24, an early biomarker for HIV infections. Real world applications of the new technique were demonstrated with the commercially available Perkin Elmer Alliance kits greatly improving their sensitivity limits, thus demonstrating that the sensitivity and reproducibility of this approach are as good as those of high-end, sensitive immunoassays. The results of this study pave the way for the development of a highly sensitive screening protocol based on any fluorescent nanoparticle based immunoassay.
\end{abstract}

Received 7th June 2018

Accepted 14th August 2018

DOI: $10.1039 / \mathrm{c} 8 \mathrm{na00019k}$

rsc.li/nanoscale-advances antigen-antibody interaction for analyte detection and use of a fluorophore as a signal transducer (in fluorescence based immunoassays $^{6}$ ). Traditional immunoassays have been suffering from inherent problems such as longer incubation durations, slow assay kinetics and the requirement for extremely accurate handling of reagents. ${ }^{7}$ Moreover, in fluorescence based immunoassays, the sensitivity is solely dependent on the quantum yield of the reporting fluorophore and the performance of the detection methodology applied in the immunoassay. ${ }^{8}$ Thus, the optimization of sensitivity and the duration of the test is limited to these features of immunoassays.

The past decade has seen significant improvement in the sensitivity of HIV-1 p24 antigen assays. This improvement can be attributed to implementation of immune complex disruption methods, using more efficient lysis buffers, and incorporation of tyramide-mediated boosting techniques in the assay. ${ }^{9}$ Another sensitive method, real-time immuno-Polymerase Chain Reaction (immuno-PCR), has inbuilt amplification methods which can detect 1000 HIV-1 RNA copies, or 40 attograms of HIV-1 p24 antigen, per reaction. ${ }^{\mathbf{1 0}}$ However, all these improvements of detection sensitivity increase the complexity of testing which makes their deployment in resource limited settings unfeasible. Unlike these methods, immunoassays have no such amplification step that can be performed during the detection which can increase the analyte concentration to detectable limits. However, with a simple modification of the immunoassay method, amplification of the fluorescence signal strength
${ }^{c}$ Laboratory of Molecular Virology, Center for Biologics Evaluation and Research

(CBER), Food and Drug Administration, Silver Spring, MD 20993, USA

${ }^{a}$ Laboratories for Nanoscience and Nanotechnology Research, Department of Physics, Sri Sathya Sai Institute of Higher Learning, Prasanthi Nilayam, Puttaparthi 515134, India.E-mail:vrkamisetti@gmail.com

${ }^{b}$ Andhra Pradesh Medtech Zone, AMTZ, Vishakhapatnam, Andhra Pradesh 530045, India 
can be achieved, which is implementing the use of metal enhanced fluorescence. Metal enhanced fluorescence can greatly improve the sensitivity limit by one order of magnitude.

Metal-enhanced fluorescence (MEF) refers to the use of metal colloids and nanoscale metallic particles in fluorescence systems. ${ }^{11}$ It was observed that when excited fluorophores are placed in the vicinity of metal nanostructures, their emission can couple to the dipoles on those plasmonic nanostructures, which under certain conditions reradiate the emission which is amplified compared to the free space emission of the fluorophore. ${ }^{12}$ Importantly, fluorophore-metal systems are created due to the high efficiency of coupling of the signal to the surface plasmon modes on the metal nanoparticle systems. ${ }^{13}$ This, when combined with their high efficiency to radiate, results in high fluorescence quantum yields and reduced emission lifetimes. Hence, MEF has attracted a lot of attention which has resulted in its implementation in specific analytical and microscopy applications where increased brightness and enhanced photostability are highly favored. ${ }^{\mathbf{1 4}}$ The outcome is that we see an amalgamation of enhancement factor coupled with enhanced photostability in the detection method. ${ }^{15}$ The major advantage is that the immunoassays with fluorophores possessing low quantum yield can make use of the metal enhanced fluorescence phenomenon to boost their sensitivity.

In this work, we describe a novel application of MEF through a single step modification to a conventional nanoparticle based immunoassay using gold nanoparticles which has resulted in a ten-fold increase in its sensitivity. We have modified the existing europium nanoparticle sandwich immunoassay with MEF (MEF-ENIA) to achieve a sensitivity of less than $1 \mathrm{pg} \mathrm{\textrm {mL } ^ { - 1 }}$ in the detection of HIV-1 p24. Along with the application in the detection of p24 antigen, we explored how MEF enhances the detection sensitivity with a known viral load. Furthermore, real world applications were also demonstrated with regard to the commercially available Perkin Elmer Alliance kits improving their sensitivity limits. We also demonstrate that the sensitivity and reproducibility of this approach are as good as those of high-end, sensitive immunoassays and also that the method is capable of providing better results with point of care testing in resource limited settings. The results of this study can pave the way for the development of a highly sensitive screening protocol based on any fluorescent nanoparticle based immunoassay which can be greatly beneficial in clinical settings and support the control and prevention of the spread of AIDS.

\section{Methods}

\subsection{Bioconjugation of europium nanoparticles (EuNPs)}

The Bioconjugation of EuNPs was carried out using the EDCNHS coupling protocol. ${ }^{16} 100 \mu \mathrm{g}$ of $200 \mathrm{~nm}$ europium nanoparticles (Thermo Scientific, USA) were dispersed in $10 \mathrm{mM}$ phosphate buffer $(\mathrm{pH}=7.4)$ and were washed in centrifugal spin columns. Nanoparticles were redispersed in Phosphate Buffer Saline (PBS). The carboxyl groups were activated with $10 \mathrm{mmol} \mathrm{L}^{-1}$ EDC (Thermo Scientific, USA) and $10 \mathrm{mmol} \mathrm{L}^{-1}$ NHS (Thermo Scientific, USA) in PBS buffer for 30 minutes. The activated particles were washed once with wash buffer. To this solution, $100 \mu \mathrm{L}$ solution of $1 \mathrm{mg} \mathrm{mL}^{-1}$ streptavidin (Scripps Lab, USA) dissolved in carbonate bicarbonate buffer $(\mathrm{pH}=9.0)$ was added. The above prepared solution was incubated at room temperature for $3 \mathrm{~h}$ following which the streptavidin conjugated nanoparticles were washed 5 times with the wash buffer.

\subsection{Immunoassay protocol}

A solution of capture antibody (ANT-152) (Prospec, USA) at a concentration of $2 \mu \mathrm{g} \mathrm{mL}{ }^{-1}$ was prepared by diluting the stock solution with carbonate-bicarbonate buffer (100 mM, pH 9.6). $55 \mu \mathrm{L}$ of this capture antibody solution was coated onto Nunc maxisorp fluorescence microplates and the plates were incubated for 24 hours at $4{ }^{\circ} \mathrm{C}$. After the incubation period, the plates were washed with wash buffer 5 times followed by washing the wells with $300 \mu \mathrm{L}$ of casein blocking buffer (Thermo Scientific, USA). The casein block buffer, whose role is to ensure that all the non-specific absorption sites are blocked, was incubated for 30 minutes at $37^{\circ} \mathrm{C}$. The next step is addition of the HIV-1 p24 antigen. The stock solution of HIV-1 p24 (Virogen, USA) was diluted with block buffer to prepare different concentrations of the antigen. $100 \mu \mathrm{L}$ of the antigen was added to each well and incubated at $37^{\circ} \mathrm{C}$ with shaking for 1 hour. Following the incubation step, all the wells were washed 5 times with wash buffer. In the consequent step, $100 \mu \mathrm{L}$ of biotinylated detector antibody (Perkin-Elmer, USA) was added to each well and incubated at $37^{\circ} \mathrm{C}$ for 60 minutes. This step completes the formation of this antibody-antigen-antibody sandwich complex in the well, to which $100 \mu \mathrm{L}$ of streptavidin conjugated europium nanoparticles (EuNPs) were added and left to incubate again for 30 minutes at $37^{\circ} \mathrm{C}$ with shaking. A final round of washing was performed 5 times with wash buffer to avoid nonspecific interactions and reduce the background noise. The microplate was placed in the SpectraMax M5 microplate reader and the fluorescent signal intensities from the sandwich immunocomplexes were measured in a time resolved mode with excitation at $340 \mathrm{~nm}$ and emission at $615 \mathrm{~nm}$. Subsequently, $100 \mu \mathrm{L}$ of $150 \mathrm{~nm}$ gold nanoparticles (Sigma-Aldrich) were added to obtain an amplified signal through metal enhanced fluorescence. Once again, the signal was measured and recorded using the SpectraMax M5 reader. All the experiments were performed in triplicate. Samples with signal-toblank ratios (SBR) equal to or greater than 1.5 were considered positive for HIV-1 p24.

\subsection{Preparation of plasma samples with a known viral load}

Prior to the preparation of the plasma samples for the immunoassay, they were confirmed to be HIV negative or HIV positive using standard HIV detection kits. Further, the viral load was calculated using the PCR techniques. To prepare the test samples, $2 \mu \mathrm{L}$ of plasma was diluted in $188 \mu \mathrm{L}$ of casein block buffer and $10 \mu \mathrm{L}$ of $10 \%$ aqueous solution of Triton X-100 was added. The samples were diluted by one order of magnitude, sequentially, to obtain the desired concentrations of the viral loads to be tested with the conventional immunoassay and MEF-ENIA. 


\section{Results}

\subsection{Choice of plasmonic nanoparticles}

Gold nanoparticles were chosen as the signal enhancers because of their wide range of size dependent surface plasmon resonance which allows their application in different nanoparticle based immunoassays employing different fluorophores. ${ }^{17}$ Due to their high chemical stability, even small gold nanoparticles are observed to be stable indefinitely. The surface modifications are simpler and conjugations are easily done because the surface chemistry of gold is well studied allowing easy surface modification and binding of biomolecules. ${ }^{\mathbf{1 8 , 1 9}}$ Additionally, there are many methods of synthesis of gold particles and shells of a variety of shapes. ${ }^{20}$ Thus, gold nanoparticles were chosen as the signal enhancers for our experiments.

The first choice that was to be made was the size of gold nanoparticles to be used in the immunoassay. The fluorescence enhancement is controlled by the degree of spectral overlap between the plasmon resonance and the excitation/emission wavelengths of the fluorophore. ${ }^{21,22}$ Specifically, the emission wavelength of europium nanoparticles must be slightly blueshifted from the plasmon resonance of gold nanoparticles for maximum enhancement to occur. ${ }^{23}$ There are a wide range of sizes available for the gold nanoparticles but keeping in mind the conditions for spectral overlap and the emission wavelength of EuNPs as $615 \mathrm{~nm}$, the ideal size for gold nanoparticles that have a plasmon oscillation frequency, which is blue shifted, is $150 \mathrm{~nm}$ which has a plasmon resonance wavelength of nearly $630 \mathrm{~nm}$. The absorption and the photoluminescence spectra are presented in Fig. 1(a) and (b). Moreover, if there is any change in the features of gold nanoparticles due to agglomeration, the enhancement factor would drastically decrease as the spectral overlap condition will not be met for MEF to occur. Thus, neither larger nor smaller particles can be used in this study and the size has to be around $150 \mathrm{~nm}$. Thus, we chose $150 \mathrm{~nm}$ gold nanoparticles for all our subsequent studies.

An important feature in the choice of the gold nanoparticles in this study is the surface functionalization. The $150 \mathrm{~nm}$ gold nanoparticles (Sigma-Aldrich) which are used in this study are citrate ligand functionalized with a shelf life of 6 months. The citrate ligands act as the capping agents which prevent the agglomeration of the nanoparticles. Apart from the role of a capping agent, the citrate groups also prevent non-specific absorption between gold nanoparticles and EuNPs. This can be attributed to the weak electrostatic interactions between streptavidin on EuNPs and citrate on gold nanoparticles, which are negligible to cause any non-specific absorption. ${ }^{24}$ This ensured that the gold nanoparticles did not negatively affect the sensitivity of the immunoassay.

The next important parameter that affects the signal enhancement levels is the concentration of the gold nanoparticles that are being added. To assess the correct concentration of the gold nanoparticles, the EuNP immunoassay was performed for a fixed concentration of p24 with different concentrations of gold nanoparticles $(150 \mathrm{~nm})$ being added before the measurement of the signal. The signal was measured with and without the gold nanoparticles to see the percent change that occurs in the signal response once the gold nanoparticles are added. The detailed protocol of the immunoassay is presented in Fig. 2. Fig. 3 schematically shows the increase in the signal strength after addition of gold nanoparticles. The percent change for different concentrations of gold nanoparticles is plotted in Fig. 4.

We observed that there is a quenching phenomenon which occurs at higher concentrations of gold nanoparticles. As the concentration is reduced the percent quenching decreases and an amplification is observed. The enhancement of the signal increases till it peaks at the concentration of $0.01 \mathrm{mg} \mathrm{mL}^{-1}$ after which it drops again. The quenching observed happens because of the narrow gap between the dipoles and gold nanoparticles at higher concentrations. In metal-enhanced fluorescence, a fluorophore must be at a distance of several nanometers from the metallic film or the nanoparticle to avoid quenching of the fluorescence through non-radiative energy transfer with the metal. ${ }^{25}$ At the appropriate distance, quenching is reduced significantly and the enhanced electric field of the surface plasmons improves the excitation rate and reduces the lifetime of the fluorophore, with the net effect of higher fluorescence intensity. ${ }^{\mathbf{8} 26}$ Theoretically, this appropriate distance is the average distance between the particles in the ensemble. But, it cannot be measured experimentally due to the dynamic and
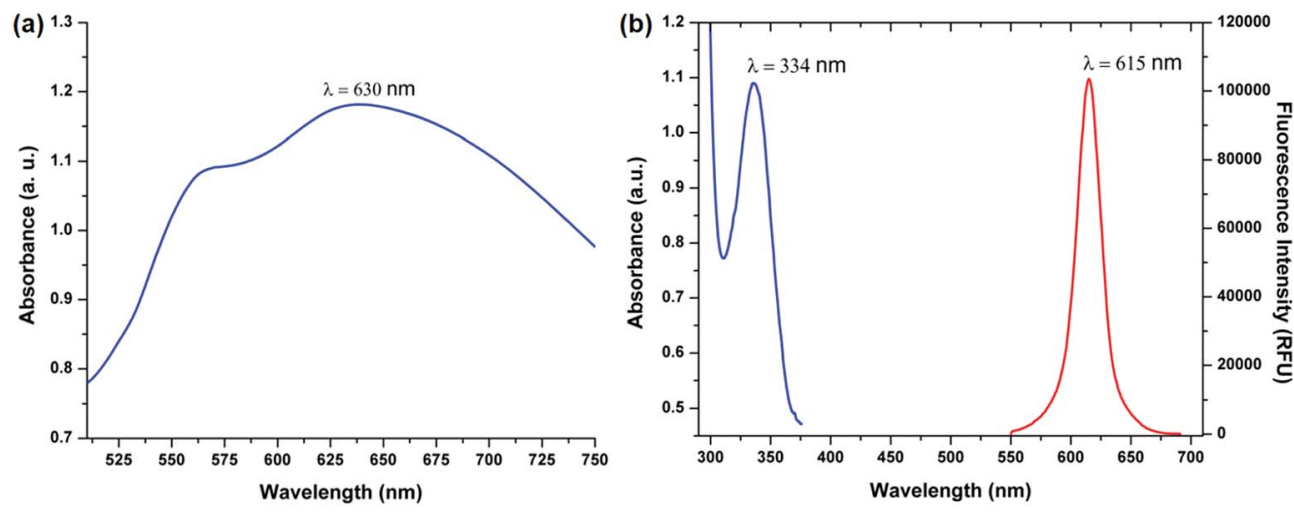

Fig. 1 (a) Absorbance spectra of $150 \mathrm{~nm}$ gold nanoparticles. (b) Absorbance and photoluminescence spectra of $200 \mathrm{~nm}$ europium nanoparticles. 
Coating the wells with capture antibodies

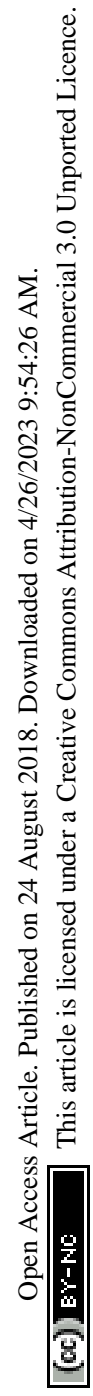
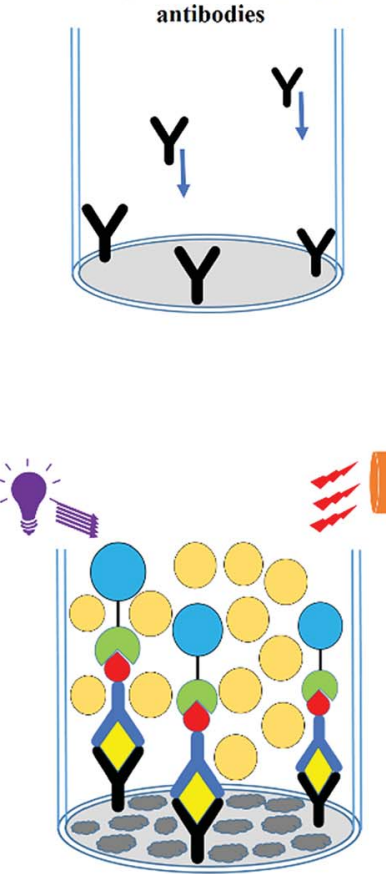

Measurement of Metal enhanced fluorescence signal from the wells
Addition of block buffer to

the well

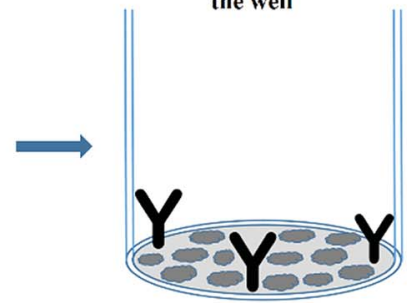

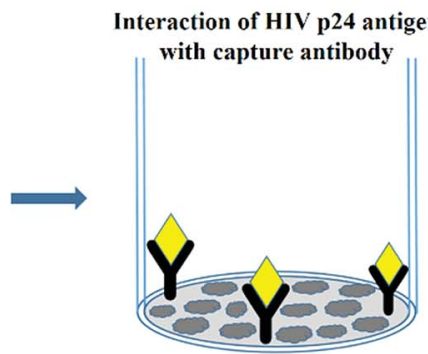

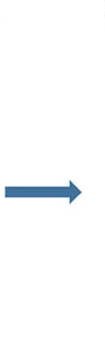

Biotinylated detector antibody interaction with the antigen
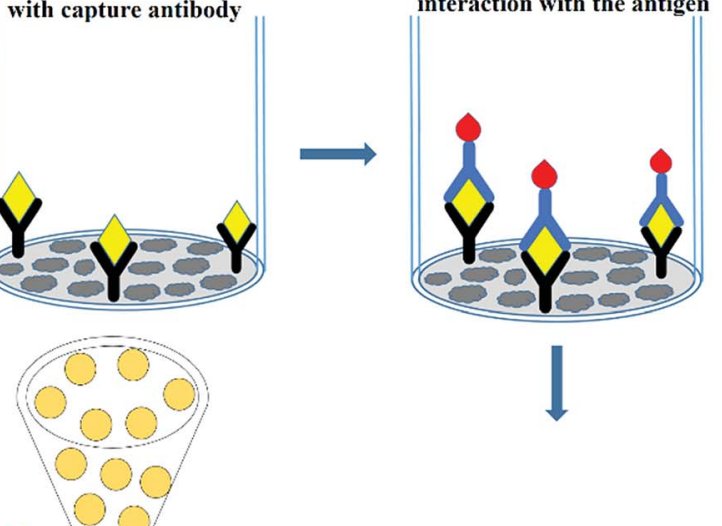

$\downarrow$

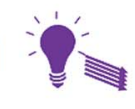

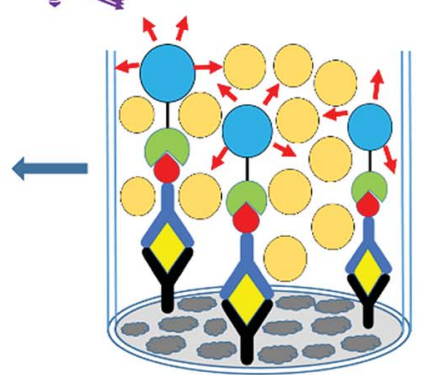

Interaction of fluorescence emission With gold nanoparticle resulting in MEF

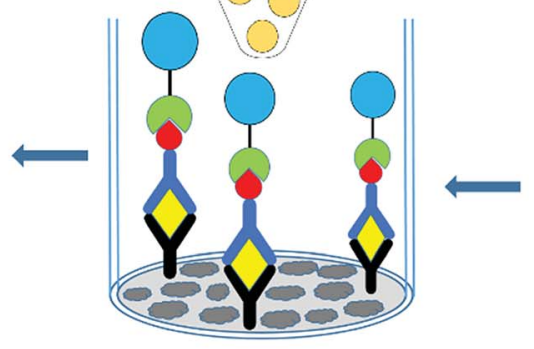

Addition of gold nanoparticles for signal enhancement step

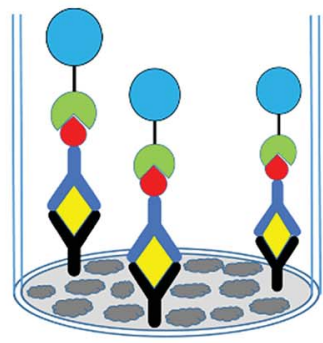

Addition of EuNP-SA to the sandwich complex through streptavidin-biotin interaction

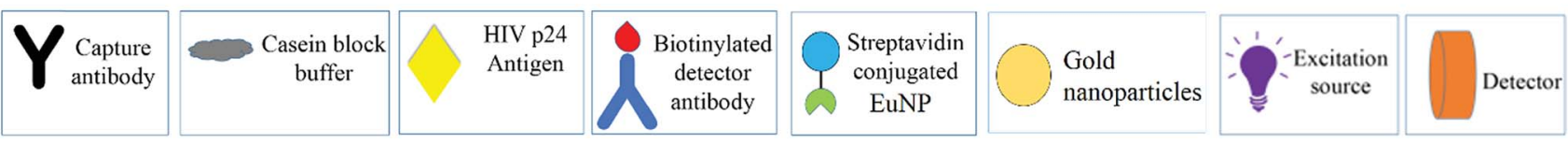

Fig. 2 Schematic illustration of the protocol involved in metal enhanced fluorescence based ENIA.
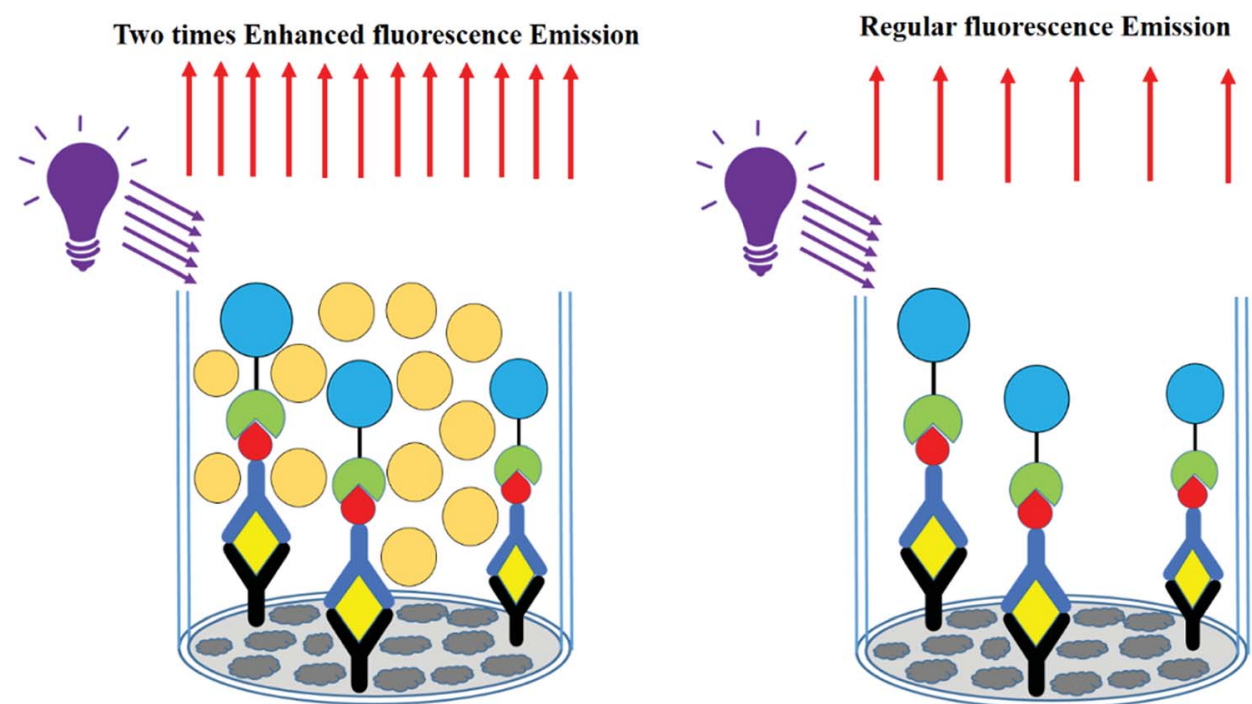

Metal Enhanced Fluorescence EuNP Immunoassay

Conventional EuNP Immunoassay

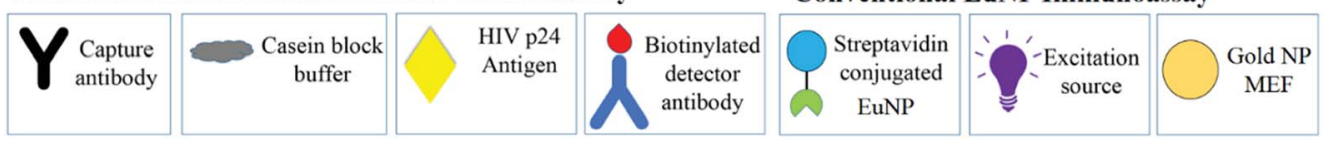

Fig. 3 A schematic comparing the fluorescence emissions in the conventional immunoassay and metal enhanced fluorescence based ENIA. 


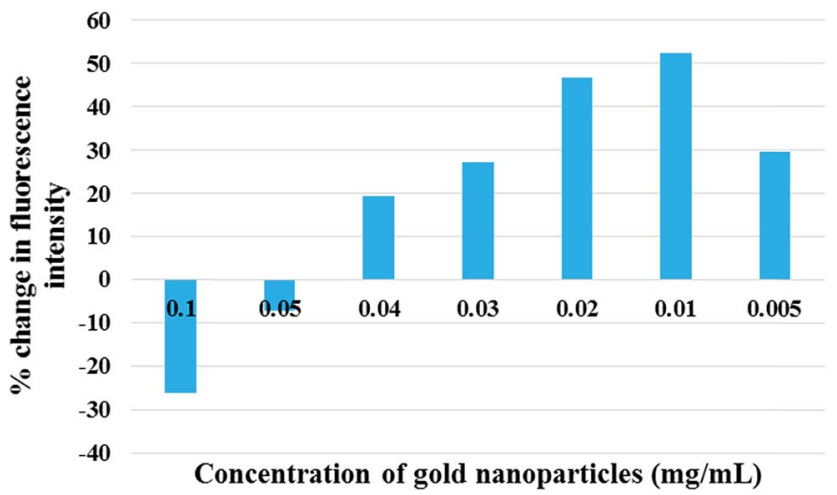

Fig. 4 The dependence of percent change in signal intensity on the concentration of gold nanoparticles.

chaotic movement of particles in solution. The only way to maintain it is via the optimization of the concentration of the nanoparticles. As understood from Fig. 4, the increased concentration of gold nanoparticles causes overcrowding due to the small volume available for the particles to move around, which reduces the distance between the gold nanoparticles and the europium fluorophores. This crowding reduces as the concentration reduces because the lower concentration implies more degree of movement for the particles. The nanoparticles, when at an optimal concentration, are at the optimal distance to interact with the emitting dipoles and maximum enhancement occurs. ${ }^{27}$ As the concentration is further reduced the number of nanoparticles available to enhance the signal falls drastically and thus, the signal response also falls. Thus, the enhancement of fluorescence is a delicate balancing act between the optimal distance between the nanoparticle and the fluorophore, and the optimal number of nanoparticles.

\subsection{Application of metal enhanced fluorescence to ENIA}

With the parameters optimized, the MEF was applied to europium nanoparticle immunoassays for the detection of HIV-1 p24 in clinical samples. In order to test the difference in the immunoassay results by adding the gold nanoparticles for MEF, a standard ENIA was performed with varying concentration of HIV-1 p24. The conventional immunoassay procedure was followed and the signal was measured directly from the antibodyantigen-antibody sandwich. This step was immediately followed by the enhancement step where $100 \mu \mathrm{L}$ of $0.01 \mathrm{mg} \mathrm{mL}^{-1}$ gold nanoparticles $(150 \mathrm{~nm})$ were added per well and the signal was recorded again.

The results are very promising, indicating probable application of MEF in immunoassays in future. A two-fold signal enhancement was observed when we added gold nanoparticles to the plate wells. Not only was the signal enhanced, the sensitivity of the assay also increased two-fold when it came to the lower end of the dynamic range of detection as seen in Fig. 5. The ENIA which could detect up to $4 \mathrm{pg} \mathrm{mL}^{-1}$ of p24 with standard parameters of the assay was able to detect $1 \mathrm{pg} \mathrm{mL}^{-1}$ as seen from Fig. 6 . The new limit of detection was calculated to be $0.19 \mathrm{pg} \mathrm{mL}^{-1}$ which was much lower than the limit of

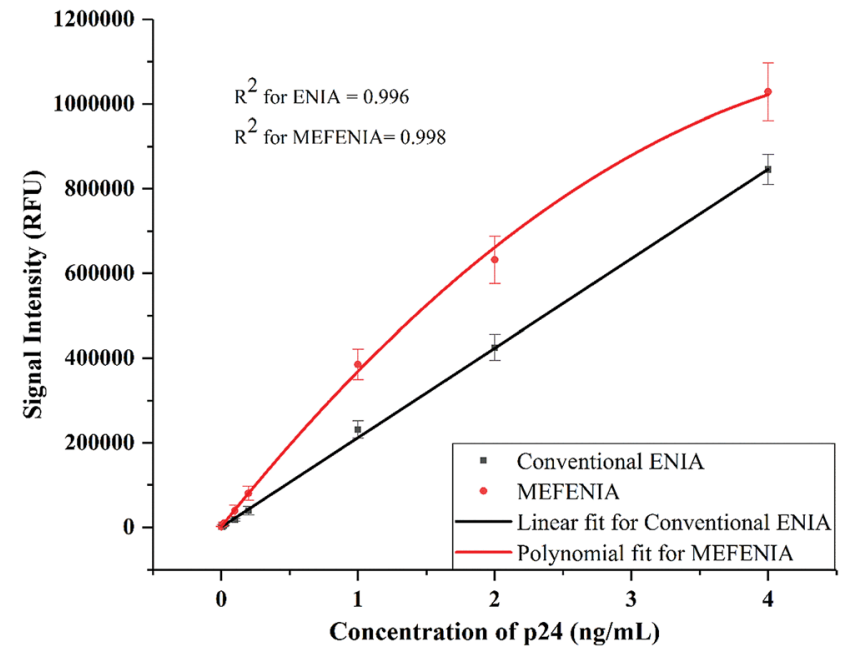

Fig. 5 Comparison of calibration curves of conventional ENIA (black line) and MEF-ENIA (red line).

detection of the conventional assay which was around $1.80 \mathrm{pg}$ $\mathrm{mL}^{-1}$. Hence, we see a ten-fold increase in the limit of detection. The reason for the multifold increase of sensitivity can be explained by the ability of metal nanoparticles to reject the far field noise and specifically amplify the near field signal. ${ }^{28}$ The signal was found to be stable over the triplicate tests and also substantially higher than the blank signal measured. The signal intensity increase that was observed was tested across multiple repetitions of the assay. Thus, we could confirm the signal enhancement which happened after addition of gold nanoparticles.

The nonlinear behavior of the MEF-ENIA standard curve can be understood by the saturation of photon-plasmon coupling within the metal nanoparticles at higher p24 concentration. Due to the increasing photon count at higher concentrations of analyte, the number of photons that couple with the plasmon of

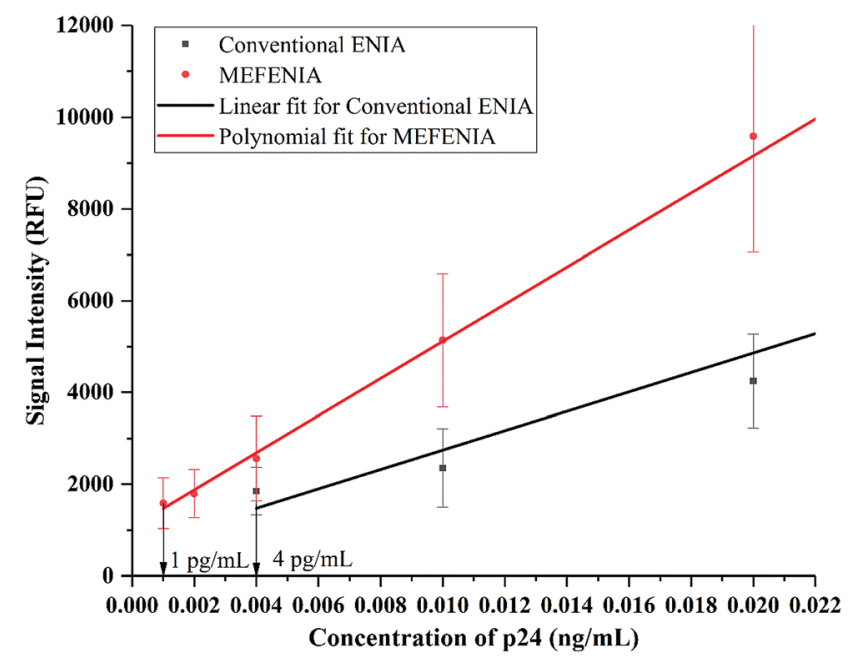

Fig. 6 The calibration curves with a resolved axis indicating the lower limit of the dynamic range of detection achieved with conventional ENIA (black line) and MEF-ENIA (red line). 
the gold nanoparticles increases. After a certain concentration, there is an excess of photons which results in the inability of the gold particles to interact with the excess photons. Consequently, this causes a fall in the signal enhancement resulting in the nonlinear nature of the standard curve.

The limit of detection has improved from $1.8 \mathrm{pg} \mathrm{mL}^{-1}$ to 0.19 pg $\mathrm{mL}^{-1}$ which is a ten-fold increase. While the absolute change in the value may seem insignificant, the increase in sensitivity can be of more prominence when it can be observed for less sensitive assays. Thus, the increase in sensitivity is a great performance improvement which can be further applied to any other immunoassay if the right size of gold nanoparticles is chosen and spectral overlap conditions are met.

\subsection{Application of MEF to improve the detection limit of samples with a known viral load}

The next step was to check the application of MEF in dealing with other real world limitations like the detection of low viral numbers. The detection of a low number of viral copies has been a problem with immunoassays and has been traditionally dealt with PCR based techniques. Thus, in order to improve the sensitivity of the assay to detect low copy numbers, MEF can be a possible modification to the immunoassay protocols that can boost the sensitivity by an order of magnitude. The immunoassay was performed with clade B sample, which was

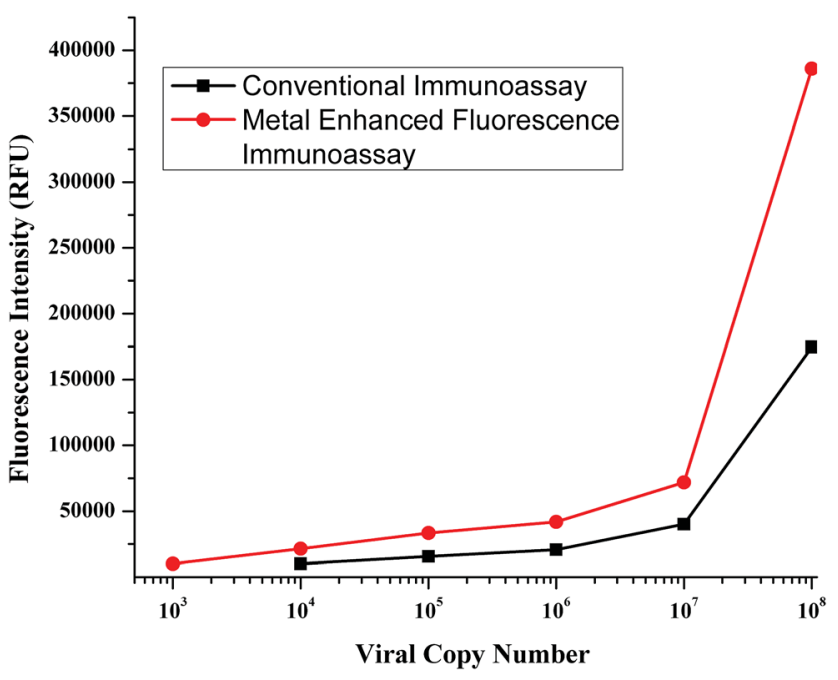

Fig. 7 Calibration curves obtained for the conventional immunoassay (black curve) and MEF-ENIA immunoassay (red curve) in the detection of p24 in samples with a known viral load. sequentially diluted to certain orders of magnitude, to test the application of MEF methodology. The test was performed and the signal was measured following which the enhancement step was performed by addition of gold nanoparticles. The signals were recorded and plotted for comparison.

A striking improvement in the sensitivity was observed when the gold nanoparticles were added for signal enhancement. As seen from Fig. 7, the sensitivity has been improved by an order of magnitude. The previous detection limit was reported to be $10^{4}$ which has improved to $10^{3}$. This order of magnitude increase was reported with a standard incubation period of one hour. If this metal nanoparticle enhancement is performed with higher incubation durations, then maybe the limit can be expected to be further lowered. This leads to a possibility of having an immunoassay protocol which is extremely sensitive which can pick up markers in the early stages of infection.

\subsection{Application of MEF to improve the sensitivity of commercially available Perkin Elmer Alliance kits}

With an increased sensitivity observed in a variety of applications, one of the real problems which can be tackled is increasing the sensitivity of detection with antibodies of commercially available kits which are reported to have lower sensitivity compared to ANT-152 (ProSpec) which are used in ENIA. The Perkin Elmer Alliance kit has been reported to have a sensitivity of $12.5 \mathrm{pg} \mathrm{mL^{-1 }}$ in an enzyme substrate based reporting format, which may not be enough for the sensitive detection of p24 in early stages of infections. Thus, the Perkin Elmer commercially available antibody coated kit was used to check the sensitivity through ENIA, instead of ELISA, and the sensitivity was found to be $8 \mathrm{pg} \mathrm{mL^{-1 }}$. To further increase the sensitivity of this commercially available kit, the MEF step was added while performing the immunoassay and the signals were recorded before and after the addition of gold nanoparticles. The blank measurements were made with PBS which also acted as a negative control. The signal intensities were then divided by the blanks to obtain the signal to blank ratios. The recorded signals are tabulated in Table 1.

There was a considerable improvement in the signal despite the low signal strength due to lower concentration. While the signal to blank ratio, as seen from the table, was higher for 10

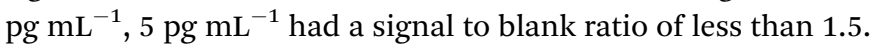
In this case, this meant that the sample cannot be conclusively characterized as positive, whereas, once the MEF step was performed and the signal was measured again, we could see an increase in the signal intensity and also the signal to blank ratio

Table 1 Comparison of signal strengths of conventional ENIA and MEF-ENIA with capture antibodies from a Perkin Elmer kit Alliance kit

\begin{tabular}{|c|c|c|c|c|}
\hline \multirow{2}{*}{$\begin{array}{l}\text { Concentration of } \\
\text { HIV-1 p } 24\left(\mathrm{pg} \mathrm{mL}{ }^{-1}\right)\end{array}$} & \multicolumn{2}{|c|}{ Signal intensity (relative fluorescence units) } & \multicolumn{2}{|c|}{ Corresponding signal to blank ratio } \\
\hline & Conventional ENIA & MEF ENIA & Conventional ENIA & MEF ENIA \\
\hline 5 & 1554 & 1996 & 1.41 & 1.82 \\
\hline 10 & 3258 & 5577 & 2.97 & 5.08 \\
\hline Blank wells & 1102 & 1178 & - & - \\
\hline
\end{tabular}


Table 2 Comparison of various europium nanoparticle based immunoassays for the detection of HIV-1 p24 antigen with MEF-ENIA

\begin{tabular}{llll}
\hline & & $\begin{array}{l}\text { Limit of detection } \\
\left(\mathrm{pg} \mathrm{mL}^{-1}\right)\end{array}$ & Reference \\
\hline 1 & Microfluidic chip & 5.0 & 29 \\
2 & Conventional ENIA & 3.3 & 30 \\
3 & Conventional ENIA & 1.8 & Present work \\
4 & Bio-barcode amplification & 0.5 & 4 \\
5 & MEF-ENIA & 0.19 & Present work
\end{tabular}

increased to 1.8 , which makes the sample HIV positive. Thus, the sensitivity of Perkin Elmer Alliance kits can be increased by almost two fold by addition of gold nanoparticles. This can imply further increase in sensitivity with other more sensitive nanomaterials or upon optimization of immunoassay parameters.

Due to the enhancement factor, MEF can boost the sensitivity of immunoassays and help in realizing much lower sensitivities with pre-existing assay methodologies like conventional ENIA. Table 2 presents the comparison of limits of detection of other assays with that of MEF-ENIA. Clearly, addition of the MEF method to conventional ENIA has led to achieving sub picogram sensitivity in assays which were less sensitive previously.

Based on the results achieved in this novel modified assay, we have once again opened up the possibility of using immunoassays for ultrasensitive detection of p24 with minimal modification to the assay format. This one step modification is much simpler and effective compared to other modification steps involved in bio-barcode amplification and microfluidics. Thus, MEF based assays can be a useful and simple tool for application in resource limited settings where there is a need for simpler and sensitive techniques with minimal infrastructure.

\section{Conclusion}

We have modified the europium nanoparticle sandwich immunoassay with metal enhanced fluorescence through gold nanoparticles to achieve a sensitivity of less than $1 \mathrm{pg} \mathrm{mL} L^{-1}$ in the detection of p24 which is an early biomarker for HIV infections. The results are very promising indicators of the application of MEF in immunoassays in future because a tenfold enhancement of the limit of detection was observed when we added gold nanoparticles to the plate wells. Not only was the signal enhanced, the sensitivity of the assay was also increased manifold when it came to the lower end of the linear range of detection as seen in Fig. 6. The ENIA which could

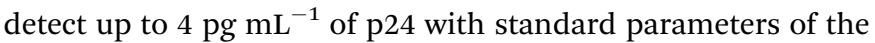
assay was able to detect $1 \mathrm{pg} \mathrm{mL}^{-1}$ which is the new sensitivity now observed for ENIA. The new limit of detection was calculated to be $0.19 \mathrm{pg} \mathrm{mL}^{-1}$, which was much lower than the limit of detection of the conventional assay, which was around 1.80 $\mathrm{pg} \mathrm{mL}^{-1}$. With a ten-fold increase in the limit of detection, we could surpass the picogram barrier to achieve a sub-picogram level sensitivity. The reason for this multifold increase of sensitivity can be explained by the ability of MEF to reject the far field noise and specifically amplify the near field signal. The signal was found to be stable over the triplicate tests and also substantially higher than the blank signal measured. The amplification of the signal that was observed was tested across multiple repetitions of the assay. Thus, we could confirm the signal enhancement which happened after addition of gold nanoparticles.

Real world applications of the new technique were demonstrated with the commercially available Perkin Elmer Alliance kits greatly improving their sensitivity limits, thus, demonstrating that the sensitivity and reproducibility of this approach are as good as those of any other high-end, sensitive immunoassay and also that the method is capable of providing better results with point of care testing in resource limited settings. The results of this study pave the way for the development of a highly sensitive screening protocol based on any fluorescent nanoparticle based immunoassay which can be greatly beneficial in clinical settings and support the control and prevention of the spread of AIDS.

\section{Disclaimer}

The findings and conclusions in this report are those of the authors and do not necessarily represent the views of the Food and Drug Administration, U.S. Department of Health and Human Services.

\section{Statement of informed consent}

We confirm that informed consent was obtained after the nature and possible consequences of the studies were explained.

\section{Data and materials availability}

All data needed to evaluate the conclusions in the paper are present in the paper and/or the ESI. Additional data related to this paper may be requested from the authors.

\section{Author contribution}

K. V. and I. K. H. conceived the study; A. K., H. G. M. K., and L. A. A. C. designed the experiments and analyzed the data. A. K. synthesized and characterized the nanoparticles. A. K. and C. S. M. performed the computational simulations. A. K. and L. A. A. C. performed the assay. A. K., K. V., and I. K. H. wrote the manuscript.

\section{Conflicts of interest}

The authors declare that they have no competing interests. 


\section{Acknowledgements}

All authors are grateful to Bhagawan Sri Sathya Sai Baba for his constant inspiration and guidance. Aditya Kurdekar thanks DST for their support through the DST INSPIRE Fellowship program, Ministry of Science and Technology, Government of India. The authors thank the Sri Sathya Sai Institute of Higher Medical Sciences for providing the plasma samples for clinical analysis.

\section{References}

1 The Global HIV/AIDS Epidemic, HIV.gov, available at: https://www.hiv.gov/federal-response/pepfar-global-aids/ global-hiv-aids-overview, 2017, accessed: 14th October 2017.

2 M. S. Cohen, C. L. Gay, M. P. Busch and F. M. Hecht, The detection of acute HIV infection, J. Infect. Dis., 2010, 202, S270-S277.

3 S. Tang, et al., Nanoparticle-Based Biobarcode Amplification Assay (BCA) for Sensitive and Early Detection of Human Immunodeficiency Type 1 Capsid (p24) Antigen, J. Acquir. Immune Defic. Syndr., 2007, 46, 231-237.

4 S. Tang and I. Hewlett, Nanoparticle-based immunoassays for sensitive and early detection of HIV-1 capsid (p24) antigen, J. Infect. Dis., 2010, 201, S59-S64.

5 R. M. Lequin, Enzyme immunoassay (EIA)/enzyme-linked immunosorbent assay (ELISA), Clin. Chem., 2005, 51, 24152418.

6 A. Bange, H. B. Halsall and W. R. Heineman, Microfluidic immunosensor systems, Biosens. Bioelectron., 2005, 20, 2488-2503.

7 I. A. Hemmilä, Applications of Fluorescence in Immunoassays, Wiley, New York, 1991.

8 J. R. Lakowicz, Topics in Fluorescence Spectroscopy, Kluwer Academic Publishers, New York, 2002, vol. 4.

9 J. Schupbach, et al., Sensitive Detection and Early Prognostic Significance of p24 Antigen in Heat-Denatured Plasma of Human Immunodeficiency Virus Type 1-Infected Infants, $J$. Infect. Dis., 1994, 170, 318-324.

10 J. M. Barletta, D. C. Edelman and N. T. Constantine, Lowering the detection limits of HIV-1 viral load using real-time immuno-PCR for HIV-1 p24 antigen, Am. J. Clin. Pathol., 2004, 122, 20-27.

11 C. D. Geddes, Metal-enhanced fluorescence, Phys. Chem. Chem. Phys., 2013, 15, 19537.

12 J. R. Lakowicz, Radiative decay engineering: biophysical and biomedical applications, Anal. Biochem., 2001, 298, 1-24.

13 K. Aslan, et al., Metal-enhanced fluorescence: an emerging tool in biotechnology, Curr. Opin. Biotechnol., 2005, 16, 5562.

14 C. D. Geddes, I. Gryczynski, J. Malicka, Z. Gryczynski and J. R. Lakowicz, Metal-enhanced fluorescence: potential applications in HTS, Comb. Chem. High Throughput Screen., 2003, 6, 109-117.
$15 \mathrm{~J}$. R. Lakowicz, et al., Advances in surface-enhanced fluorescence, J. Fluoresc., 2004, 14, 425-441.

16 P. Yousefpour, F. Atyabi, R. Dinarvand and E. VasheghaniFarahani, Preparation and comparison of chitosan nanoparticles with different degrees of glutathione thiolation, Daru, 2011, 19, 367-375.

17 Gold Nanoparticle Properties, available at: http:// www.cytodiagnostics.com/store/pc/Gold-NanoparticleProperties-d2.htm, 2017, accessed: 14th October 2017.

18 J. C. Love, L. A. Estroff, J. K. Kriebel, R. G. Nuzzo and G. M. Whitesides, Self-assembled monolayers of thiolates on metals as a form of nanotechnology, Chem. Rev., 2005, 105, 1103-1169.

19 A. Ulman, Formation and Structure of Self-Assembled Monolayers, Chem. Rev., 1996, 96, 1533-1554.

20 N. L. Rosi and C. A. Mirkin, Nanostructures in biodiagnostics, Chem. Rev., 2005, 105, 1547-1562.

21 H.-P. Poirier-Richard, M. Couture, T. Brule and J.-F. Masson, Metal-enhanced fluorescence and FRET on nanohole arrays excited at angled incidence, Analyst, 2015, 140, 4792-4798.

22 Y. Chen, K. Munechika and D. S. Ginger, Dependence of Fluorescence Intensity on the Spectral Overlap between Fluorophores and Plasmon Resonant Single Silver Nanoparticles, Nano Lett., 2007, 7, 690-696.

23 K. A. Willets and R. P. Van Duyne, Localized Surface Plasmon Resonance Spectroscopy and Sensing, Annu. Rev. Phys. Chem., 2007, 58, 267-297.

24 G. V. Presnova, et al., Streptavidin conjugates with gold nanoparticles for visualization of single DNA interactions on the silicon surface, Biochem. Suppl. Ser. B Biomed. Chem., 2014, 8, 164-167.

25 Y. Fu, J. Zhang and J. R. Lakowicz, Largely Enhanced SingleMolecule Fluorescence in Plasmonic Nanogaps Formed by Hybrid Silver Nanostructures, Langmuir, 2013, 29, 27312738.

26 J. R. Lakowicz, Radiative decay engineering 5: metalenhanced fluorescence and plasmon emission, Anal. Biochem., 2005, 337, 171-194.

27 K. A. Kang, J. Wang, J. B. Jasinski and S. Achilefu, Fluorescence Manipulation by Gold Nanoparticles: From Complete Quenching to Extensive Enhancement, $J$. Nanobiotechnol., 2011, 9, 16.

28 J. Lee, S. Lee, M. Jen and Y. Pang, Metal-Enhanced Fluorescence: Wavelength-Dependent Ultrafast Energy Transfer, J. Phys. Chem. C, 2015, 119, 23285-23291.

29 J. Liu, et al., Development of a microchip europium nanoparticle immunoassay for sensitive point-of-care HIV detection, Biosens. Bioelectron., 2014, 61, 177-183.

30 M. K. Haleyur Giri Setty, et al., Novel Time-Resolved Fluorescence europium Nanoparticle Immunoassay for Detection of Human Immunodeficiency Virus-1 Group O Viruses Using Microplate and Microchip Platforms, AIDS Res. Hum. Retrovir., 2016, 32, 612-619. 